\title{
Trends for Tuberculosis Treatment Outcomes, New Sputum Smear Positive Patients in Kwekwe District, Zimbabwe, 2007-2011: A Cohort Analysis
}

\author{
Meggie Gabida1, Mufuta Tshimanga1, Milton Chemhuru², Notion Gombe1, \\ Donewell Bangure ${ }^{1^{*}}$ \\ ${ }^{1}$ Department of Community Medicine, University of Zimbabwe, Harare, Zimbabwe \\ ${ }^{2}$ Provincial Medical Directorate, Midlands Province, Ministry of Health and Child Welfare, Gweru, Zimbabwe \\ Email: "bangured@yahoo.com
}

Received 27 October 2015; accepted 29 November 2015; published 3 December 2015

Copyright (C) 2015 by authors and Scientific Research Publishing Inc.

This work is licensed under the Creative Commons Attribution International License (CC BY).

http://creativecommons.org/licenses/by/4.0/

(c) (i) Open Access

\begin{abstract}
Introduction: Tuberculosis remains a public health problem in Zimbabwe. Understanding the treatment outcomes is an important proxy indicator on the performance of the tuberculosis program. This study analyzed treatment outcomes of new sputum smear positive tuberculosis patients in Kwekwe district. Materials and Methods: A retrospective records review of new sputum smear positive tuberculosis patients registered in the district tuberculosis register in Kwekwe was conducted. Treatment outcomes were categorized according to the national tuberculosis control program and multivariate logistic regression model was used. $P$ values less than 0.05 were considered statistically significant. Results: From a total of 1115 new sputum smear positive tuberculosis patients, cure rate ranged from $40.8 \%$ to $62.8 \%$ and death rate of patients decreased from $11(8.0 \%)$ in 2007 to $17(5.3 \%)$ in $2011(p=0.016)$. However, defaulter rate increased from $10(7.3 \%)$ in 2007 to $30(9.3 \%)$ in 2011 . In multivariate logistic model, HIV positive tuberculosis patients were more likely to experience (adjusted RR $=1.84,95 \%$ CI: $1.10-3.08$ ) unfavorable treatment outcome when compared to negative counterparts. Urban residents were also at risk 1.91 (95\% CI: 1.14 - 3.20) unfavorable outcome compared to rural residents. Conclusion: The cure rate was low (ranged from $40.8 \%$ to $62.8 \%$ ) and the defaulter rate needed urgent attention. The district should conduct defaulter tracing and follow up.
\end{abstract}

\section{Keywords}

Sputum Smear Positive, Treatment Outcome, Tuberculosis, Kwekwe Zimbabwe

\footnotetext{
"Corresponding author.
}

How to cite this paper: Gabida, M., Tshimanga, M., Chemhuru, M., Gombe, N. and Bangure, D. (2015) Trends for Tuberculosis Treatment Outcomes, New Sputum Smear Positive Patients in Kwekwe District, Zimbabwe, 2007-2011: A Cohort Analysis. Journal of Tuberculosis Research, 3, 126-135. http://dx.doi.org/10.4236/itr.2015.34019 


\section{Introduction}

Tuberculosis (TB) is a serious public health problem in Zimbabwe. World Health Organization (WHO) defines tuberculosis as an infectious bacterial disease caused by Mycobacterium tuberculosis, which most commonly affects the lungs. It is transmitted from person to person via droplets from the throat and lungs of people with the active tuberculosis. In healthy people, infection with Mycobacterium tuberculosis often causes no symptoms [1]. Tuberculosis is treatable with a six-month course of antibiotics under the Directly Observed Treatment Short Course (DOTS) [2].

Globally in 2010, there were 8.8 million incident cases of TB, and WHO estimates TB to be among the leading causes of death and disability. The effects are hardly felt among the economically active segment of the world's population [2] [3].

The international targets for tuberculosis control, framed within the United Nations' Millennium Development Goals, are to ensure that by 2015 the global TB incidence rate is declining and the global TB prevalence and death rates for 1990 are halved [4]. These targets are to be achieved by implementing WHO's Stop TB Strategy (founded on the core DOTS strategy. This strategy was launched as a coherent strategy that enables existing achievements to be sustained while effectively addressing the remaining constraints and challenges [4]. It underpins efforts to strengthen health system, alleviate poverty and advance human rights. It has a vision of a world free of TB and targets to eliminate TB as a public health problem by 2050 [1] [4].

The global burden of tuberculosis (TB) is highest in the Sub-Saharan Africa and can be attributed to the high prevalence of human immunodeficiency virus infection (HIV) which is known to increase the risk of developing TB [5]. Zimbabwe, which ranks 17th among the 22 tuberculosis high burden countries as of 2009, had a TB incidence rate of 762 cases per 100,000 population and an HIV co-infection prevalence of above $75 \%$ in 2008 . TB remains one of the main public health threats in Zimbabwe and the key activities in the control of tuberculosis are early diagnosis of individuals with TB and their correct and efficient treatment in such a way that no drug resistance is created [3] [5] [6].

There are three priority strategies that are fundamental to TB prevention and control in Zimbabwe. One of the priorities involves health programs that are directed at decreasing morbidity and mortality. These strategies involves the identification and treatment of persons who have active TB, active case finding and screening of vulnerable persons who have been in contact with TB patients, those working in dusty environments, HIV infected and health community workers (HCWs) to determine whether they have TB infection or disease and provide them with appropriate prophylactic treatment. Also screening of high risk populations to detect persons infected with $M$. Tuberculosis and whose early treatment would prevent further spread of the infection to the uninfected persons is among the priorities [3] [7].

In sub Saharan Africa, the reported challenges for the TB control programs are human immunodeficiency virus (HIV) co-infection and drug resistance [7]. TB-HIV co-infection has been shown by several studies to be a risk factor for progression to TB disease and high mortality has been reported among HIV-infected TB patients [8].

Zimbabwe adopted the recommended WHO DOTS policy package in 1994. By end of 2002 DOTS coverage was assumed to be $100 \%$ (geographically). DOTS have been recommended by international tuberculosis organizations, and have been shown to be effective in achieving a high successful treatment rate, from $86 \%$ to $96.5 \%$. DOTS also lead to significant reductions in the frequency of primary drug resistance, acquired drug resistance and relapse [9].

Kwekwe district covers a total population of 316,058 (one fifth of midlands province). The district has a total of 42 health facilities (three hospitals) and all offers TB services according to the national TB guidelines. In the district, tuberculosis control program services are available through a decentralized network of primary health care facilities that provide DOTS. Every health facility captures diagnosed TB cases into the facility TB register and the information is consolidated at district level into the district TB register [3]. The 2010 TB records showed that an average of $72 \%$ of all TB cases are HIV positive and more than $99 \%$ of all TB patients were offered testing and counseling services [10].

The 2010 tuberculosis cohort analysis showed that the province performed below expected WHO 2011 to 2015 revised targets of $87 \%$ cure rate, less than 5\% death rate and defaulter rate and zero percent treatment failure and transfer outs [11]. The major contributing poor performing district was Kwekwe district with a cure rate of $61 \%$, death and defaulter rate of $12 \%$ and $14 \%$ respectively. Treatment outcome results serve as a proxy of 
the quality of TB treatment provided by a health care system and in Midlands Province, a five year period trend analysis has never been carried out. This study aimed to analyze trends of TB treatment outcomes of new sputum smear-positive patients for each year of cohorts in Kwekwe district over a period of five years.

\section{Methods}

\subsection{Study Design and Setting}

We conducted a retrospective cohort analysis of all sputum smear positive TB patients diagnosed and treated according to the national TB control program guidelines. The cases were registered from two diagnostic and treatment centres in Kwekwe district. Kwekwe district is one of the eight districts in the midlands province and it serves a total population of 315,336 people. There are two TB diagnostic centres namely Kwekwe General Hospital and Silobela Hospital (designated district hospital). At the hospitals, there is the Directly Observed Therapy (DOT) clinic where diagnosis of pulmonary tuberculosis is followed by the examination of sputum by acid fast bacilli (AFB) smear microscopy method. Chest radiographs are also used to support the diagnosis. Patients diagnosed with tuberculosis are then referred to the DOT clinic where they are registered and treated according to the National Tuberculosis Control program guidelines. Also according to the guidelines all tuberculosis cases are offered provider initiated HIV testing and counselling.

\subsection{Data Collection}

A review of the District TB register with information of all TB patients registered from the two diagnostic and treatment centers from 2007-2011 was conducted. These registered documents contained basic information such as patient's age, sex, address, tuberculosis type, type of patient, HIV tested results from 2010 and treatment outcome. All diagnosed TB cases are recorded in the health facility TB register and receive treatment according to the national tuberculosis control program (NTP).

The outcome variables of interest were the proportion of new sputum smear-positive cases cured, treatment failure, died while on treatment, transferred out and treatment completed against World Health Organization (WHO) targets. Other variables of interest that were included in the analysis were: age measured in years, patient sex (male or female), and HIV status (positive vs. negative). HIV test among TB cases is mandatory/recommended in Zimbabwe.

The work was conducted in compliance with the Institutional Review Boardrequirements of the Health Studies Office and permission to carry out the study was sought from Provincial Medical Director-Midlands province and the District Medical Officer Kwekwe district.

\subsection{Operational Definitions}

According to the Zimbabwe tuberculosis national guidelines the following clinical cases and definitions for TB treatment outcomes were used [3].

New Smear positive TB case is a patient who has never had treatment for TB or who has taken anti-TB drugs for less than one month

Treatment outcome was divided into six categories according to the national TB control program guidelines: cured (a patient who was smear positive at diagnosis, who was smear-negative at the end of the last month of treatment and on at least one previous occasion), treatment completed (any patient who was smear-positive at diagnosis and had completed treatment but in whom smear examination results were not available at the end of treatment) died (any patient who died irrespective of the cause of death during the course of treatment) treatment failure (any patient who remained or became positive at the end of the fifth month or later during treatment), treatment defaulter (any patient who has interrupted treatment two consecutive months or more after the date of the last attendance during the course of treatment) and transfer-out (a patient who was transferred to another treatment center and whose treatment outcome is unknown).

\subsection{Defined Outcome Variables}

In the final logistic regression model, favorable treatment outcome was the combination of Cured and Treatment completed while deaths, defaulted, treatment failure and transfer out were defined as unfavorable treatment outcome. 


\subsection{Statistical Analysis}

All records of TB patients from 2007 to 2011 were included in this study. Data was double entered into Epi Info version 3.5.1 then checked and edited for inconsistencies. Data was then exported to SPSS version 16 for analysis. Results were expressed as absolute frequencies (n) and percentages (\%). We used proportions with 95\% confidence intervals and Chi-square test to compare different groups. Bivariate and multivariate logistic regression was carried out for selected socio-demographic and clinical risk factors including age, sex, place of residence and HIV status of TB patients. Since data was determined to be missing at random, a complete case analysis was conducted. $\mathrm{P}$ values of less than 0.05 were considered statistically significant.

\section{Results}

Completeness of the District TB register was above 95\%. A total of 4105 TB cases were registered from 2007 to 2011. Of all the registered TB cases, 90\% were new cases while 9\% were re-treatment cases and $1 \%$ other cases. According to diagnosis, 336 (8.2\%) were extra pulmonary tuberculosis cases, 1741 (42.4\%) were pulmonary sputum smear-negative (PTB-), 738 (18.0\%) were pulmonary TB sputum not done and 1290 (31.4\%) were sputum smear-positive (PTB+).

\subsection{Demographic Characteristics of New Sputum Smear Positive TB Patients}

Table 1 shows the general characteristics of the new sputum smear positive TB patients $(n=1115)$. Of these 501 (44.9\%) were females while 614 (55.1\%) were males. The age group 24 - 35 years had the majority of TB patients, 325 (35.5\%). The majority of patients were from urban populations 787 (70.7\%) while 310 (27.1\%) was from rural populations, $0.5 \%$ and $0.9 \%$ percent from mines and new farms respectively. Of all new sputum smear-positive TB cases tested for HIV, 78.9\% were HIV positive and $21.1 \%$ were HIV negative respectively.

Table 1 Characteristics of study population $(n=1115)$, new sputum smear positive tuberculosis patients, Kwekwe district, 2007-2011.

\subsection{Treatment Outcome Trends}

Among the sputum smear positive tuberculosis patients analyzed for the period under review for each year of cohorts, those cured was 78 (56.9\%) in 2007, 75 (40.8\%) in 2008, 126 (64.3\%) in 2009, 129 (46.9\%) in 2010 and $203(62.8 \%)$ in 2011. The death rate of patients decreased over the period under review from $11(8.0 \%)$ in 2007, 25 (13.6\%) in 2008, 22 (11.2\%) in 2009, 22 (9.8\%) in 2010 and 17 (5.3\%) in 2011. However, the default rate was increased across the years of cohorts with a peak reached in 2010. It increased from 10 (7.3\%) in 2007, $11(6.0 \%)$ in 2008, 8 (4.1\%) in 2009, $33(12.0 \%)$ in 2010 and 30 (9.3\%) in 2011. It is worth noting that another outcome termed "not located" was frequently recorded among the 2008 cohort, 11 (6.0\%), $3(1.1 \%)$ in 2010 and $5(1.5 \%)$ in 2011.

Table 2 Treatment outcomes by year, new sputum smear positive tuberculosis patients, Kwekwe district, 2007-2011.

Figure 1 shows that males had a trend to be more likely to experience death than females as an outcome $(\mathrm{RR}=1.03,95 \% \mathrm{CI}=0.68-1.56)$. In terms of outcome by place of residence, the 2007 cohort showed that two (6.7\%) and nine (8.9\%) in 2007, zero $0 \%$ and $22(14 \%)$ in 2008, four (6.9\%) and $17(12.4 \%)$ in 2009, six (8.6\%) and $21(10.3 \%)$ in 2010 and four (3.1\%) and 13 (6.9\%) in 2011, rural and urban residents died respectively. Therefore across the five cohorts, urban residents had a trend of more likely to die, compared to their rural counterparts $(\mathrm{p}=0.010)$.

\subsection{TB/HIV Co-Infection}

Comprehensive information on TB/HIV treatment documentation commenced in 2010. Seventy seven (46.1\%) HIV positive TB patients were cured in 2010 and 123 (59.3\%) were cured in 2011. The proportion of deaths decreased from $10.8 \%$ in 2010 to $5.3 \%$ in 2011 while defaulter rate decreased from $10.2 \%$ in 2010 to $9.2 \%$ in 2011 . There was no treatment failure recorded in 2010, while in 2011, 1.5\% was recorded. On the other hand, HIV positive TB patients were more likely to have an unfavorable treatment outcome $(\mathrm{RR}=2.07,95 \% \mathrm{CI}$; 1.12 3.81). 
Table 1. Characteristics of study participants $(n=1115)$ new sputum-smear positive tuberculosis patients, Kwekwe district, 2007-2011.

\begin{tabular}{|c|c|c|}
\hline \multirow[b]{2}{*}{ Characteristic } & \multicolumn{2}{|c|}{ New Sputum Smear-Positive $(\mathrm{PTB}+)$ N = 1115} \\
\hline & Frequency & Percent \\
\hline \multicolumn{3}{|l|}{ Age Group Years } \\
\hline $0-4$ & 10 & 0.9 \\
\hline $5-14$ & 25 & 2.2 \\
\hline $15-24$ & 133 & 11.9 \\
\hline $25-34$ & 396 & 35.5 \\
\hline $35-44$ & 325 & 29.1 \\
\hline $45-54$ & 127 & 11.4 \\
\hline $55-64$ & 63 & 5.7 \\
\hline$>65$ & 36 & 3.2 \\
\hline \multicolumn{3}{|l|}{ Sex } \\
\hline Females & 501 & 44.9 \\
\hline Males & 614 & 55.1 \\
\hline \multicolumn{3}{|l|}{ HIV Status } \\
\hline Negative & 100 & 21.6 \\
\hline Positive & 373 & 78.9 \\
\hline \multicolumn{3}{|l|}{ Residence } \\
\hline Mines and Farms & 16 & 1.4 \\
\hline Rural & 310 & 27.9 \\
\hline Urban & 787 & 70.7 \\
\hline
\end{tabular}

Table 2. Treatment outcomes by year, new sputum smear positive tuberculosis patients, Kwekwe district, 2007-2011.

\begin{tabular}{|c|c|c|c|c|c|c|}
\hline \multirow{2}{*}{$\begin{array}{l}\text { Treatment } \\
\text { Outcomes }\end{array}$} & \multicolumn{5}{|c|}{ Years } & \multirow{2}{*}{$\begin{array}{c}\text { Total } \\
1115(\%)\end{array}$} \\
\hline & $2007137(\%)$ & $2008184(\%)$ & 2009196 (\%) & 2010275 (\%) & 2011323 (\%) & \\
\hline Cured & 78 (56.9) & 75 (40.8) & $126(64.3)$ & 129 (46.9) & 203 (62.8) & $611(54.8)$ \\
\hline Died & $11(8.0)$ & 25 (13.6) & $22(11.2)$ & $22(9.8)$ & $17(5.3)$ & $97(8.7)$ \\
\hline Defaulted & $10(7.3)$ & $11(6.0)$ & $8(4.1)$ & $33(12.0)$ & $30(9.3)$ & $92(10.0)$ \\
\hline Failure & $0(0.0)$ & $0(0.0)$ & $4(2.0)$ & $1(0.4)$ & $5(1.5)$ & $9(0.9)$ \\
\hline Transfer out & $5(3.6)$ & $6(3.3)$ & $7(3.6)$ & $7(3.6)$ & $13(4.0)$ & $38(3.4)$ \\
\hline $\begin{array}{l}\text { Treatment } \\
\text { completed }\end{array}$ & $33(24.1)$ & $56(30.4)$ & 29 (14.8) & $70(26.0)$ & 49 (15.5) & $196(17.6)$ \\
\hline *Not Located & $0(0.0)$ & $11(6.0)$ & $0(0.0)$ & $3(1.1)$ & $5(1.5)$ & $19(1.7)$ \\
\hline $\begin{array}{c}\text { Treatment } \\
\text { Success }\end{array}$ & 81 & 71.2 & 79.1 & 72.4 & 78.3 & 72.4 \\
\hline
\end{tabular}

Figure 2 shows the age distribution of new sputum smear-positive TB patients in relation to HIV status. The highest proportions of the patients with TB HIV co-infection was in the age group 35 - 44 years accounting for $36.2 \%$ of the patients followed by the 25 - 34 years (35\%).

In the final multivariate logistic model, the proportion recorded as having unfavorable outcome varied by age group, HIV status and place of residence (Table 3). The risk of unfavorable treatment outcome was 2.76 (95\% CI: 1.40 - 5.44) times higher among TB patients older than 65 years. In addition, HIV positive TB patients were 


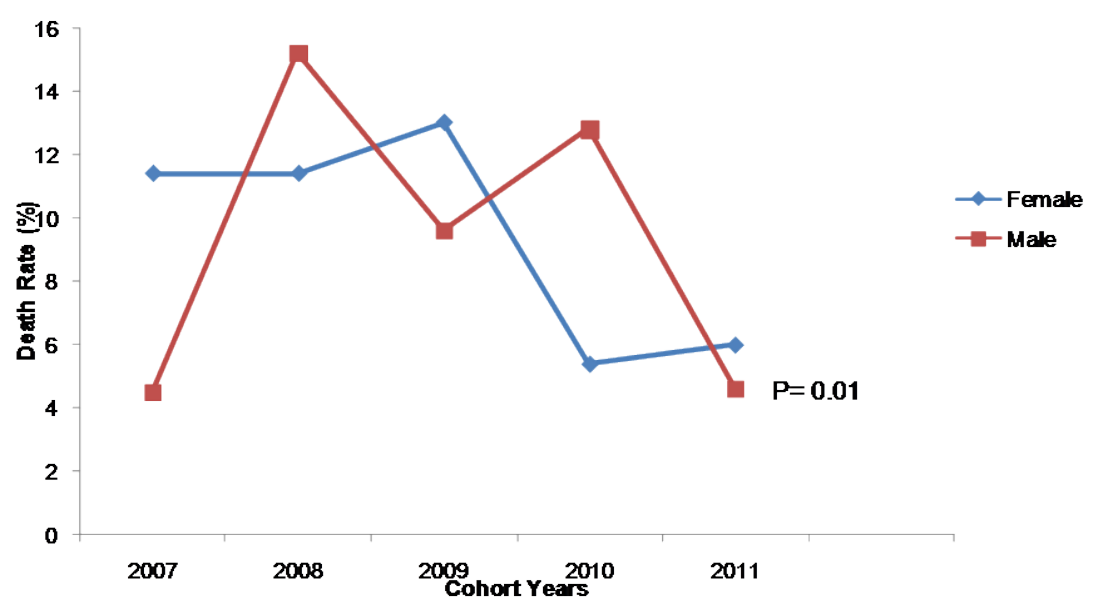

Figure 1. Trend of deaths by sex, new sputum smear-positive TB patients, Kwekwe district, Zimbabwe, 2007-2011.

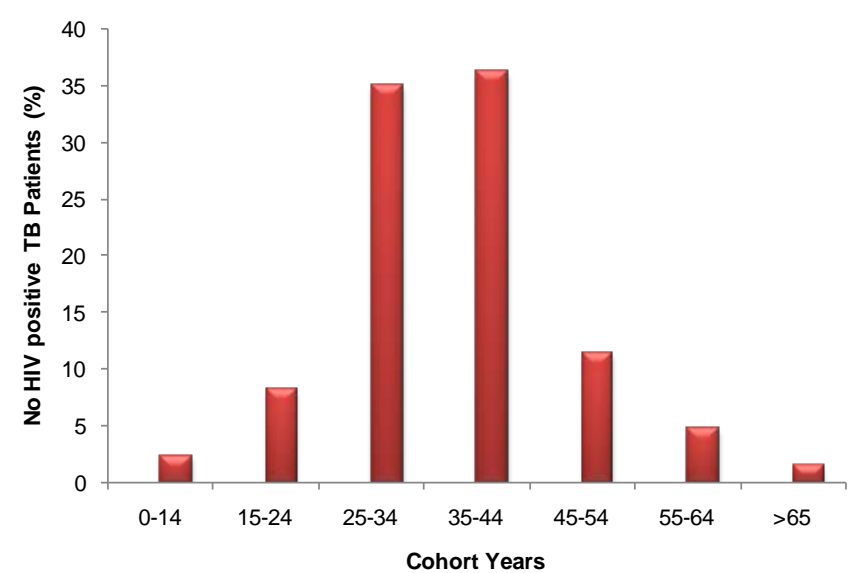

Figure 2. Age distribution for TB/HIV co-infected sputum smear positive TB patients, Kwekwe district, Zimbabwe, 2010-2011.

Table 3. Adjusted OR for treatment outcomes of sputum smear-positive tuberculosis patients, Kwekwe district, 2007-2011.

\begin{tabular}{|c|c|c|c|}
\hline \multirow[t]{2}{*}{ Characteristics } & \multicolumn{3}{|c|}{ Treatment Outcome } \\
\hline & N* Unfavorable n (\%) & COR (95\% CI) & AOR (95\% CI) \\
\hline \multicolumn{4}{|l|}{ Age (years) } \\
\hline $0-64$ & 66 (10.0) 591 (90.0) & 1.00 & 1.00 \\
\hline$>65$ & $11(55.0) 9(45.0)$ & $2.82(1.43-5.56)$ & $2.76(1.40-5.44)$ \\
\hline \multicolumn{4}{|l|}{ Sex } \\
\hline Female & 46 (11.2) 366 (88.8) & 1.00 & 1.00 \\
\hline Male & 101 (23.7) 325 (76.3) & $1.10(0.89-1.37)$ & $1.10(0.89-1.58)$ \\
\hline \multicolumn{4}{|l|}{ HIV Status } \\
\hline Negative & $14(14.0) 86(86.0)$ & 1.00 & 1.00 \\
\hline Positive & 91 (24.8) 276 (75.2) & $1.80(1.07-3.02)$ & $1.84(1.10-3.08)$ \\
\hline \multicolumn{4}{|l|}{ Residence } \\
\hline Rural & 37 (14.0) 227 (86.0) & 1.00 & 1.00 \\
\hline Urban & 78 (10.2) 689 (89.8) & 1.87 (1.12 - 3.15) & $1.91(1.14-3.20)$ \\
\hline
\end{tabular}


more likely to experience (adjusted RR $=1.84,95 \% \mathrm{CI}$ : 1.10 - 3.08) unfavorable treatment outcome when compared to negative counterparts. Urban residents were also at risk 1.91 (95\% CI: 1.14 - 3.20) of unfavorable outcome compared to rural residence. Sex did not show any statistically significant association with unfavorable treatment outcome in multivariate analysis.

\section{Discussion}

An analysis of treatment outcomes is of paramount importance to the national TB control program. Most importantly, treatment outcomes of sputum smear positive TB cases are among the priority strategies to TB prevention and control as they are highly infectious and thus should complete treatment and be tested at end of treatment to ensure that they are cured.

Our study found that cure rate of the five cohorts from 2007 to 2011 which ranged from $40.8 \%$ to $64.3 \%$ is lower than the WHO Target of $87 \%$ (updated target 2011-2015). The low cure rate can be due to high defaulter rate that ranged from $4.0 \%$ to $12.0 \%$, treatment completed rate of $15.5 \%$ to $30.4 \%$ and death rate of $5.3 \%$ to $13.7 \%$. Therefore further investigations are needed on these outcomes. Similar findings were observed elsewhere in Africa. In a study conducted in Malawi, treatment outcomes among the 4003 sputum smear positive PTB cases was $72 \%$ treatment completed, $20 \%$ death rate, $4 \%$ defaulter rate and $2 \%$ transfer out [12] Cure rate was greatly affected by the proportion that completed treatment. However, it is important to note that the proportions among each cohort that completed treatment was declining over the years from 2007-2011 ( $p=0.026)$. This is a positive achievement to the TB control program since this shows that a greater proportion of the sputum smear positive TB cases are being monitored and sputum is collected after treatment completion to confirm that indeed the individuals are cured. However much still needs to be done in the district to ensure that all sputum for smear-positive TB patients are collected and tested at 5months and at completion of DOT as per national TB control program guidelines.

The low cure rate and treatment success observed in our study can also be due to the fact that our DOT Clinic is facility based. A study conducted in Tanzania reported treatment success of $81 \%$ in community based DOT and $70 \%$ in patients under facility-based DOT [13]. Thus facility based DOTs should be backed by a strong community component especially on defaulter tracing as well as counseling to improve adherence to treatment.

The defaulter rate in this study was double the WHO International Target of 5\% and it was increasing across the years. It has been documented in other settings that counseling, sustained supervision, home visits and health education have been used successfully as interventions to reduce defaulter rates of tuberculosis patients [14]. As observed from the dataset, the defaulter rate could have been increased due to poor patient tracing as indicated by the documentation of another outcome that was termed not located which is not consistent with TB program guidelines. This is evidence that follow up of patients as well as guidelines adherence in the district are poor. This has negative impacts on the TB control program as treatment defaulters are more likely to develop MDR TB. Thus our findings indicate that there is need to strengthen defaulter tracing and implement interventions that may reduce defaulter rate of tuberculosis patients in Kwekwe District. A study by Datiko et al., (2009) reported that the use of health extension workers was effective in increasing treatment success and tracing defaulters [15].

This study also showed that deaths among sputum smear- positive PTB patients were decreasing across the years. This can be attributed to the provider initiated HIV testing in our setting where TB cases are encouraged to be tested and initiated on antiretroviral therapy for TB/HIV co-infected patients. In our study $86.5 \%$ and $73.6 \%$ in 2010 and 2011 respectively of the TB patients tested were HIV positive. Our findings suggest that TB/HIV collaboration in the district is effective as since 2010, there was a marked decline in deaths among TB patients. The finding is consistent with findings by Girdrdi et al., 2001 where they reported that concurrent administration of ART and anti-tuberculosis treatment in TB/HIV co-infected patients have been associated with improved survival [16]-[18]. On the other hand, TB/HIV co-infection was highest among the 25 - 44 years age group. This may be because this age group is economically and sexually active.

In our study unfavorable outcome was significantly higher among patients above 65 years of age, urban residents and those HIV positive. Our study finding concurs with findings by Tessema et al., 2009 where they observed that as age increased the death rate of patients was steadily increased. Another study conducted in Tigray Region, Northern Ethiopia found that unsuccessful treatment outcome was significantly higher among patients older than 40 years of age [12] [19] [20]. Another study in Thailand showed that an age above 60 years was significantly correlated with treatment interruption and treatment failure [21]. Older age has been reported in many studies as a risk factor for death [19]-[21]. This may be, because older individuals are challenged by many de- 
terminants of health including low socio-economic status than the younger generation. This age group is also physiologically deteriorating and cannot fight many infections and some of them are less able to reach health facilities [16] [22]. Thus older age requires close monitoring to reduce deaths among TB patients.

HIV positive TB patients were shown by several studies to be associated with unsuccessful treatment outcomes which include treatment interruption and death. Another study conducted in Ethiopia also showed that HIV positive patients are more likely to default treatment than the HIV negative counterparts [17] [23]. These results however, are not in agreement with a study conducted by Berhe et al., 2012 in the Northern Region of Ethiopia, where they found no association between HIV status and unsuccessful outcome [14]. This can be explained by the fact that our study was a cohort analysis of new sputum smear positive TB patients only and was comparable unlike the study in the Tigray Region where it looked at sputum positive TB patients including new and retreatment cases.

We also observed higher deaths in urban populations compared to rural. The possible explanation may be that sputum smear positive TB is highly infectious, and in some urban areas, people are densely overcrowded per $\mathrm{m}^{2}$ compared to rural residents. It is also possible that most of the houses in some urban areas are poorly ventilated and not to forget that sick rural folks migrate to urban areas in seek of better health care at advanced stages of illness.

Our study found that rural residents were more likely to default treatment compared to those in urban. This can be attributed to the fact that, in rural areas physical access to health facilities is a challenge. Even though treatment is offered for free in public institutions, there are other in-direct costs that may lead patients to default treatment in rural settings. Our findings are consistent with a study conducted in Southern Ethiopia, where physical access to health care services was associated with treatment interruption [24].

Data from this study revealed that sex was not significantly associated with treatment outcome but male gender had a trend of more likely to die than females. There are several possible explanations; males have poor health seeking behavior than females and usually they seek medical attention late at advanced stage of the disease. Males also decline HIV testing and majority gets tested when critically ill. Furthermore, males often have poor adherence to treatment and default treatment compared to females [17] [23]. This Lack of association was somewhat unexpected as reports from other studies indicate these as significantly associated with unsuccessful treatment particularly male gender. The differences in our study findings with other studies can be due to a myriad of factors. These may include differences in socio demographic factors and the burden of tuberculosis in various settings. Also in this study we did not look at treatment outcomes and associated risk factors for other forms of TB. However, our finding concurs with findings by Berhe et al., 2012 where different sexes of patients did not show any statistical significant association with unsuccessful treatment outcome [16].

Our study reveals that defaults and deaths (unfavorable treatment outcomes) are still threatening the success of the TB control program. There is need to carry out further investigations to explain the factors associated with an increase in high defaulter rates in the district.

Study Limitation

The limitation of this study was that, information on HIV was only documented comprehensively from 2010 to 2011.

\section{Conclusion}

The cure rate was far below the target of $87 \%$. Deaths and default rates were above the recommended WHO target of less than $5 \%$ and were a public health concern. To improve treatment outcome in Kwekwe, we recommend defaulter tracing, support and supervision as well as continued health education to TB patients to reduce defaults.

\section{Acknowledgements}

Authors would like to thank Mrs. Mwale, the TB Coordinator and other staff members at various DOTS clinics of Kwekwe district for their unwavering support during data collection.

\section{Competing Interests}

The authors declare no competing interest. 


\section{Authors' Contributions}

MG was the primary researcher, designed, participated in data collection, conducted data analysis and prepared the first draft manuscript. MT, DB, NG and MC interpreted the results, supervised the project and reviewed the first draft manuscript. All authors read and approved the manuscript.

\section{References}

[1] World Health Organization (2010) Global TB Report. World Health Organization, Geneva

[2] World Health Organization (2009) Treatment of TB Guidelines. 4th Edition, World Health Organization, Geneva.

[3] Ministry of Health and Child Welfare (2010) Zimbabwe National Tuberculosis Control Program. National TB Guidelines. 4th Edition, Ministry of Health and Child Welfare, Gweru.

[4] World Health Organization (2012) Stop TB Partnership. The Stop TB Strategy-Building on and Enhancing DOTS to Meet to the Tuberculosis Related Millennium Development Goals. World Health Organization, Geneva.

[5] Maher, D., Harries, A. and Getahun, H. (2005) TB and HIV interaction in Sub-Saharan Africa: Impact on Patients and Programmes: Implications for Policies. Tropical Medicine \& International Health, 8, 734-742. http://dx.doi.org/10.1111/j.1365-3156.2005.01456.x

[6] Hayrapetyan, N., Mezhlumyan, H.D. and Sevoyan, M. (2012) Tuberculosis Trends in Armenia. EpiNorth, 13, 13-17.

[7] Maimela, E. (2009) Evaluation of TB Treatment Outcomes and the Determinants of Treatment Failure in the Eastern Cape Province, 2003-2005. Master’s Dissertation, University of Pretoria, Pretoria.

[8] Chennaveerappa, P.K., Siddharam, S.M., Halesha, B.R., Vittal, B.G. and Jayashree, N. (2011) Treatment Outcome of TB Patients Registered at Dots Centre in a Teaching Hospital, South India. International Journal of Biological and Medical Research, 2, 487-489.

[9] Ministry of Health and Child Welfare (2012) Midlands Province TB Report. Ministry of Health and Child Welfare, Gweru.

[10] Lawn, S.D., Myer, L., Bekker, L.G. and Wood, R. (2006) Burden of TB in an Antiretroviral Therapy Programme in Sub-Saharan Africa: Impact on Treatment Outcomes and Implications for TB Control. AIDS, 2, 1605-1612. http://dx.doi.org/10.1097/01.aids.0000238406.93249.cd

[11] Ministry of Health and Child Welfare (2011) Midlands Province Health Information Systems. Ministry of Health and Child Welfare, Gweru.

[12] Harries, A.D., Nyirenda, T.E., Banerjee, A., Boeree, M.J. and Salaniponi, F.M. (1999) Treatment Outcome of Patients with Smear-Negative and Smear-Positive Pulmonary Tuberculosis in the National Tuberculosis Control Programme, Malawi. Transactions of the Royal Society of Tropical Medicine and Hygiene, 4, 443-446. http://dx.doi.org/10.1016/S0035-9203(99)90153-0

[13] Van den Boogaard, J., Lyimo, R., Irongo, C.F., Boeree, M.J., Schaalma, H., Aarnoutse, R.E. and Kibiki, G.S. (2009) Community vs. Facility-Based Directly Observed Treatment for Tuberculosis in Tanzania's Kilimanjaro Region. International Journal of Tuberculosis and Lung Disease, 12, 1524-1529.

[14] Berhe, G., Enguselassie, F. and Aseffa, A. (2012) Treatment Outcome of Smear-Positive Pulmonary TB Patients in Tigray Region, Northern Ethiopia. BMC Public Health, 12, 537. http://dx.doi.org/10.1186/1471-2458-12-537

[15] Datiko, D.G. and Lindtjorn, B. (2009) Health Extension Workers Improve Tuberculosis Case Detection and Treatment Success in Southern Ethiopia: A Community Randomized Trial. PLoS One, 5, e5443. http://dx.doi.org/10.1371/journal.pone.0005443

[16] Tessema, B., Muche, A., Bekele, A., Reissig, D., Emmrich, F. and Sack, U. (2009) Treatment Outcome of Tuberculosis Patients at Gondar University Teaching Hospital, Northwest Ethiopia. A Five-Year Retrospective Study. BMC Public Health, 9, 371. http://dx.doi.org/10.1186/1471-2458-9-371

[17] Mitike, G., Kebede, D. and Yeneneh, H. (1997) HIV Infection and Anti Tuberculosis Drug Resistance among Pulmonary TB Patients in Tuberculosis Center, Ethiopia. East African Medical Journal, 74, 154-157.

[18] Girdrdi, E., Palmieri, F. and Cingolani, A. (1997) Changing Clinical Presentation and Survival in HIV Associated Tuberculosis after Highly Active Antiretroviral Therapy. Journal of Acquired Immune Deficiency Syndromes, 4, 326-331.

[19] Takarinda, K., Harries, A.D., Srinath, S., Sandy, C., Apollo, T. and Mugurungi, O. (2012) Treatment Outcomes of Adult Patients with new TB in Relation to HIV Status in Chitungwiza, Zimbabwe. BMC Public Health, 12, 124. http://dx.doi.org/10.1186/1471-2458-12-124

[20] Yassin, M.A., Takele, L., Gebresenbet, S., Girma, E., Lera, M., Lendebo, E. and Cuevas, L.E. (2004) HIV and TB CoInfection in the Southern Region of Ethiopia. A Prospective Epidemiological Study. Scandinavian Journal of Infectious Diseases, 9, 670-673. http://dx.doi.org/10.1080/00365540410020848 
[21] Anunnatsiri, S., Chetchotisakd, P. and Wanke, C. (2005) Factors Associated with Treatment Outcomes in Pulmonary Tuberculosis in North Eastern Thailand. Southeast Asian Journal of Tropical Medicine and Public Health, 2, 324-330.

[22] Olusoji, M. I., and Oladokun, R.E. (2011) Treatment Outcome of Newly Diagnosed Sputum Positive Adult Tuberculosis Cases in the Context of HIV. Journal of Infectious Diseases and Immunity, 10, 210-217.

[23] Vijai, S., Kumar, P., Chauhan, L.S., Rao, S.V.N. and Viadyanathan, P. (2011) Treatment Outcome and Mortality at One and Half year Follow-Up of HIV Infected TB Patients under TB Control Programme in a District of South India. PLoS One, 10, 1371. http://dx.doi.org/10.1371/journal.pone.0021008

[24] Shargie, E.B. and Lindtjorn, B. (2007) Determinants of Treatment Adherence among Smear Positive Pulmonary Tuberculosis Patients in Southern Ethiopia. PLoS One, 2, e37. 